Pre-reminder, $63.4 \%$ of targeted women completed a mammogram; this number increased to $75.4 \%$ in the post-reminder implementation; $80.6 \%$ completed a mammogram in the maintenance phases. After controlling for demographics and clinic visits, intervention women were 1.51 times more likely to complete a mammogram (CI 1.40-1.62) post-reminder implementation, compared to CG 1. The effect was maintained in 2007 (HR 1.81 [CI 1.65-1.99]). Women of other races (Asian, Pacific Island, or Native American) were less likely to complete a mammogram, as were those taking a greater number of medications. Women with a college education, who were married, or who had visited a primary care provider or gynecologist during follow-up were more likely to complete a mammogram. Conclusions: We found that a multimodal reminder system could be effectively implemented and maintained in a large health system. If widely implemented, this intervention could substantially improve community mammography screening rates. Insured patients who are sicker, or who are of Asian, Pacific Island, or Native American descent may need more support to complete screening. Future studies should address practice-based factors that assist patients in completing mammograms, as well as other factors that affect the reach and cost-effectiveness of delivering the intervention to diverse patient groups.

PS2-10:

\section{The CRN Cancer Communication Research Center}

Sarah D Madrid, MA, CRN Cancer Communication Research Center [CCRC], Kaiser Permanente Colorado; James W Dearing, $\mathrm{PhD}$, Kaiser Permanente Colorado, CRN Cancer Communication Research Center; Russell E Glasgow, $\mathrm{PhD}$, Kaiser Permanente Colorado, CRN Cancer Communication Research Center; Borsika A Rabin, PhD, Kaiser Permanente Colorado, CRN Cancer Communication Research Center; Kathleen Mazor, EdD, Meyers Primary Care Institute, University of Massachusetts Medical School; Edward H Wagner, MD, MPH, Center for Health Studies, Group Health Cooperative

We propose an integrated set of three, large posters that will describe the main components of a new research center that bridges HMORN institutions. Background: The CRN Cancer Communication Research Center (CCRC) was established in September 2008 at Kaiser Colorado Institute for Health Research. Objectives: The CCRC's objectives are to discover the most promising practice-based approaches to cancer communication and care coordination, and to disseminate those approaches. Integrated care delivery systems represent promising opportunities to study these approaches, and the CRN CCRC, with its embedded organizational focus will take advantage of the CRN as a virtual laboratory. Specific Aims: The CRN CCRC 1) leverages the existing infrastructure of the CRN to support both the discovery and dissemination of practice-based communication strategies and organizational resources; 2) supports four investigator-initiated research projects to advance communication theory and to evaluate strategies informed by theory; and 3 ) provides administrative, financial, and scientific support to new investigators, including clinicians, in the development of pilot projects, and assists in submission of broader, investigator-initiated proposals to be submitted for extramural funding. Methods: Two R01-scale investigator initiated research projects will advance and test communication theory. The first will: characterize patients' and providers' experiences communicating about errors in cancer care; investigate the health system factors that promote or inhibit effective communication; and develop, disseminate, and evaluate provider training materials and patient informational materials. The second will develop and test an intervention to decrease patient uncertainty and improve psychosocial and communicative outcomes during the period from suspicion of cancer through diagnosis and plan of care. The Center's research projects will be augmented and supported by Shared Resource Cores. The Discovery Core will identify the most promising practicebased innovations and approaches; the Dissemination Core will focus on data harmonization and applying dissemination science to effective interventions.
PS2-25:

Provider Perspectives on Essential Components of a Cancer Survivor Care Plan

Jody Jackson, RN, BSN, HealthPartners Research Foundation; Cheri J. Rolnick, PhD, MPH, HealthPartners Research Foundation; Anna Ourada, BA, American Cancer Society Midwest Division

Background: This presentation is based on efforts of the Minnesota Cancer Alliance (MCA), a coalition of more than 80 groups and individuals, to improve quality of life for cancer survivors in Minnesota. The MCA is currently developing a comprehensive and userfriendly SCP based on feedback regarding limitations of existing survivorship care plan (SCP) tools. As a first step, a needs assessment of health care providers was conducted regarding essential elements. Aims: To report on components providers wanted incorporated into the tool as well as methods to promote SCPs. Methods: In April 2008, participants (e.g., oncologists, primary care providers, nurses, social workers, community health workers) of a working session on SCPs provided feedback on desired components and strategies for communication and promotion. Results: A total of 59 health care professionals participated. Treatment given, possible side effects and a follow-up plan were considered the fundamental information needed. Participants wanted a separate psychosocial section which would include information on emotional, spiritual, sexual and economic issues (e.g., work) as well as the ability to document the survivor's support system and any social support services used. A section on family history and genetic counseling/testing was deemed important as well as a section for documentation of other comorbidities. Participants indicated that use of the $\mathrm{SCP}$ tool should begin at diagnosis and provide information for both short and long-term survival. Conclusions: Feedback obtained from providers indicated that not only were treatment history and side effects important components of a SCP, but addressing the survivor as a whole person and as part of a family system (i.e., psychosocial section, family history and genetic testing, comorbidity section) was crucial. Survivors need information along the entire continuum of cancer care and health care professionals could benefit by a user-friendly process to convey information to their patients and their caregivers. Identifying the needs of health care providers regarding the essential elements of a SCP will help meet patient needs, potentially improve patient care and may help ensure greater use of the SCP among providers.

\section{PS2-27:}

Identification of Patients With Nonmelanoma Skin Cancer Using HMO Claims Data

Melody J Eide, MD, MPH, Henry Ford Health System; Richard Krajenta, BS, Henry Ford Health System; Dayna Johnson, MPH, MSW, Henry Ford Health System; Jordan Long, Henry Ford Health System; Christine C Johnson, MPH, $\mathrm{PhD}$, Henry Ford Health System

Background: Despite the large population affected, the epidemiology of nonmelanoma skin cancer (NMSC) is understudied, and U.S. cancer registries often exclude NMSC. Secondary data analysis has been limited in that squamous cell (SCC) and basal cell carcinoma (BCC) do not have their own unique International Classification of Disease (ICD9) identifiers. Our aim was to define and compare algorithms for identifying NMSC by secondary analysis using a computerized database of a large health maintenance organization (HMO). Methods: A computerized claims database of a large HMO in Southeastern Michigan was used to identify NMSC patients who were diagnosed between January 1, 1988 to December 31, 2007. Three algorithms were examined: NMSC ICD-9 codes, Current Procedural Terminology (CPT) code for treatment of malignant NMSC, or both ICD-9 and CPT codes. A subset of charts for the overall cohort and all charts from 2007 HMOenrollee members only were reviewed to verify NMSC diagnosis. Positive predictive values were calculated. Results: Analyses of data from 1988-2007 identified NMSC in 165,000 patients using ICD-9 codes, 44,875 patients by CPT codes and 113,666 patients who had both codes. A random sample of 1275 cases were selected for chart review to verify NMSC, which was validated in $73.7 \%$ of ICD-9 identified patients, $94.6 \%$ of CPT identified patients, and $97.5 \%$ of patients identified using both codes. Data was then limited to HMO-health plan 